\title{
Valoración participativa del uso y manejo del huerto mixto
}

\author{
Participatory assessment of the mixed homegardens, use and management
}

\section{Miriam Guapucal Cuasanchir 1; Ximena Sthefania Benavides C.2; Kelly Xiomara Sinisterra3}

1. Docente, I.AF MSc., Universidad de Nariño, Pasto, Colombia, miriamgc@udenar.edu.co.

2. Ingeniera Agroforestal, Universidad de Nariño, Pasto, Colombia, eco-753@hotmail.com.

3. Ingeniera Agroforestal, Universidad de Nariño, Pasto, Colombia, kxiomara_1512@hotmail.com.

Citar: Guapucal, M., Benavides, X., Sinisterra, K. (2019). Valoración participativa del uso y manejo del huerto mixto. Revista de Ciencias Agrícolas. 36(1): 46-58. doi: http://dx.doi.org/10.22267/rcia.193601.97

Recibido: mayo 30 de 2017. Aceptado: marzo 052019.

\begin{abstract}
RESUMEN
El desconocimiento que tienen las comunidades de las especies que poseen en los huertos mixtos, así como el poco valor que le dan a los usos, llevó a desarrollar la investigación tuvo lugar en la vereda

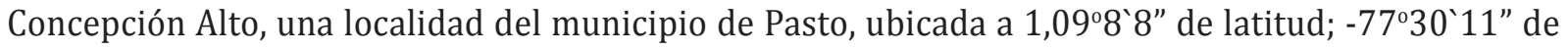
longitud y a una altura de $2520 \mathrm{msnm}$. El objeto de la investigación fue recopilar, valorar y sistematizar desde la percepción local el uso y manejo de las especies que componen los huertos mixtos, para ello se seleccionaron a 10 familias como informantes claves, siendo necesario estratificarlas por edad, para determinar en qué estrato se concentra el mayor conocimiento; para ellos se aplicó una entrevista semiestructurada. El análisis del conocimiento se hizo por medio de la metodología AKT 5 (Agroecological Knowledge Toolkit). Entre las variables evaluadas se destacan el uso específico de las especies, manejo y coeficiente de importancia, a las cuales se les realizó un análisis estadístico (prueba de t-student) complementado con un análisis de correlación complementada con una correlación entre las variables edad, escolaridad y número de frases. Los resultados indican que el conocimiento entre los estratos I $(\mu=94 \pm 27)$, II $(\mu=127 \pm 22)$ y III $(\mu=75 \pm 13)$ no mostró diferencias estadísticas significativas ( $p$-value $=0,3988>0,05$ ), es decir, que todos los estratos comparten el
\end{abstract}


mismo conocimiento, obteniendo una correlación negativa entre las variables edad y número de frases $(r=-0,31)$. En cuanto al uso que hacen de los componentes del huerto se destacan los medicinales, alimenticios, forrajeros, leña, madera, sombra y regulación hídrica. El manejo del huerto está condicionado por la experiencia, creencias y nuevos conocimientos adquiridos. En este sentido, los huertos mixtos presentes en la vereda Concepción Alto, son un reservorio de biodiversidad y conocimiento tradicional.

Palabras clave: agricultura tradicional, especies multifuncionales, valoración del conocimiento.

\begin{abstract}
The lack of knowledge communities have about species in their mixed homegardens, as well as the little value they give to their uses, led to develop the research that took place in the village of Concepción Alto, a town in the municipality of Pasto, located at $1.09^{\circ} 8^{\prime} 8$ "of latitude; $-77^{\circ} 30^{\prime} 11^{\prime \prime}$ of longitude and with 2520 masl. The purpose was to collect, evaluate and systematize the use and management of the species that make up the homegardens, 10 families as key informants were selected, being necessary to stratify them to determine which stratum had the highest knowledge; a semi-structured interview was applied. Knowledge analysis was carried out using the AKT 5 (Agroecological Knowledge Toolkit) methodology. Among the evaluated variables, the outstanding were the specific use of the species, type of management and coefficient of importance, a statistical analysis (Student $t$ test) complemented with their correlation with the variables: age, education, and the number of sentences. To analyze the information the R statistical software was used. The results indicate that the knowledge between the strata I $(\mu=94 \pm 27)$, II $(\mu=127 \pm 22)$ and III $(\mu=75 \pm 13)$ showed no statistically significant differences ( $p$-value $=0.3988>0.05)$, meaning that all sections share similar knowledge, obtaining a negative correlation between the variables of age and number of phrases $(r=-0.31)$. About the homegardens' use highlight medicine, food, fodder, fuelwood, timber, shade and water regulation. The homegarden management is conditioned by the experience, beliefs, and new acquired knowledge. In this sense, mixed homegardens in the village of Conception Alto, are a reservoir of plant biodiversity and knowledge.
\end{abstract}

Key words: traditional management, multifunctional species, assessment of knowledge.

\section{INTRODUCCIÓN}

El conocimiento local, corresponde al conjunto de saberes, creencias y costumbres que son consistentes entre si y lógicas para aquellas comunidades que la comparten (Farrington y Martin, 1988). El conocimiento local es útil para el diseño, implementación y manejo sustentable de los sistemas productivos (Muñoz et al., 2003). Uno de los sistemas tradicionales practicados en zona andina de minifundio, son los huertos mixtos o huertos agroforestales, definidos como la combinación de cultivos con árboles, en ocasiones en asociación con animales domésticos (Nair y Kumar, 2006). Ningún otro sistema agroforestal es tan diverso en cantidad de especies, variado en estructura, asociaciones $\mathrm{y}$ tan complejo en funciones (Lok, 1998).

En la vereda Concepción Alto, corregimiento de Santa Bárbara, la Universidad de Nariño ha venido promoviendo el establecimiento de los huertos mixtos, con el objetivo de fortalecer la seguridad alimentaria de las familias de la zona. La composición de estas unidades productivas es muy diversa dentro de las que se destacan especies agrícolas (hortalizas, tubérculos, raíces, cereales, legumbres), árboles 
y/o arbustos, especies aromáticas y medicinales y especies pecuarias (cuyes, conejos, cerdos y gallinas). En estos sistemas se promueve un acervo de conocimiento tradicional reflejado en su uso y manejo, lo que ha garantizado la alimentación de las familias, la conservación de recursos genéticos, complementario a esto, ha llevado a la domesticación de especies agrícolas (Sablayrolles y Andrade, 2009); sin embargo, el aprovechamiento que se puede hacer de éste conocimiento no ha tenido relevancia y su tendencia es a desaparecer por cuanto no se ha avanzado en investigaciones que lo describan, sistematicen y promuevan.

En un estudio realizado por Muñoz et al. (2008), evaluó el conocimiento local del uso y manejo de especies arbóreas y agrícolas en huertos mixtos, en dos localidades del municipio de Tumaco, utilizando la herramienta Agroecological Knowledge Toolkit(AKT5).Este programa permite crear y organizar una base de conocimiento sobre un tema particular, producto de un proceso de recopilación a través de la aplicación de diferentes herramientas de obtención de información como encuestas, entrevistas, etc., a personas clave (Dixon et al., 2001).

En este particular, el propósito de esta investigación fue recopilar, valorary sistematizar desde la percepción local el uso y manejo de las especies que componen los huertos mixtos en la vereda Concepción Alto, municipio de Pasto, seleccionando informantes claves, a los que se les aplicó una entrevista semiestructurada.

\section{MATERIALES Y MÉTODOS}

El trabajo se realizó en la vereda Concepción Alto, municipio de Pasto, localizada a 1,098` $8^{\prime \prime}$ de latitud; $-77^{\circ} 30^{\prime} 11^{\prime \prime}$ longitud y a una altura de $2520 \mathrm{msnm}$, zona caracterizada por tener una temperatura promedio de $12^{\circ} \mathrm{C}$ y la zona de vida bosque húmedo montano alto (bh-MA), (Corponariño, 2012).

La investigación se basó en la metodología propuesta por Sinclair y Walker (1999) y Dixon et al. (2001), quienes hacen uso de la herramienta informática AKT5, para la compilación y representación del conocimiento agroforestal. El estudio se desarrolló por fases, las cuales a se describen a continuación:

Fase I. Caracterización del conocimiento local sobre el uso y manejo de huertos mixtos Identificación de informantes clave. El trabajo se desarrolló con 10 familias las cuales se seleccionadas teniendo en cuenta la presencia de huertos mixtos en sus predios y la voluntad de bridar información. Los participantes en su mayoría fueron mujeres, cuyas edades oscilaban entre 23 y 60 años de edad, por ello, fue necesario estratificar por edad para determinar en qué estrato se concentra el mayor conocimiento, de acuerdo con lo propuesto por Muñoz et al. (2008), de la siguiente manera: Estrato 1. < 30 años (3 informantes); estrato 2. 31-50 años (5 informantes) y estrato $3 .>51$ años (2 informantes).

Obtención de información primaria. La información se obtuvo mediante la aplicación de entrevistas semiestructuradas complementadas con recorridos de campo. Se contemplaron dos variables como objeto de estudio: Uso específico de los componentes del huerto (medicinal, alimenticio, forraje, y otros usos como: leña, madera, cercas vivas, postes, sombra, regulación hídrica y biodiversidad) y manejo del huerto (épocas de siembra, riego, fertilización, podas, cosecha, control de plagas).

Creación de la línea base de conocimiento. Una vez obtenida la información, se procedió a construir la base de conocimiento, utilizando el programa AKT5, que permite ingresar y 
editar información por medio de un listado de términos formales, definiciones, fuentes de información, jerarquías de temas u objetos, enunciados y diagramas.

Las frases unitarias se clasificaron en: 1) atributo - valor, donde se describen procesos, objetos o acciones; 2) frases de causa, aquellas que denotan un cambio positivo o negativo; 3 ) frases de comparación, donde se confrontan términos formales y 4) frases link, donde se relacionan términos formales sencillos.

Fase II. Determinación del coeficiente de importancia de las especies (CIE). Para la valoración participativa del uso y manejo de las especies presentes en el huerto mixto, se determinó el coeficiente de importancia, considerandoelniveldeutilización,laimportancia biofísica y demanda de comercialización uso en la familia, según la ecuación desarrollada por Bentes y Gama (1999):

\section{$\mathrm{CIE}=3^{*} \mathrm{NU}+2^{*} \mathrm{IB}+\mathrm{DC}$}

Donde:

NU: Nivel de utilización, que expresa la importancia de la especie en cuanto a su funcionalidad para la familia: 3. Muy utilizada especie con tres o más usos; 2. Utilizada - especie con dos usos; 1. Poco utilizada - especie con un uso y 0 . No utilizada.

IB: Importancia biofísica, que representa la frecuencia de la especie; 3 . Alta - frecuencia (70 100\%); 2. Media - frecuencia (31 - 69\%); 1. Baja - frecuencia (1 - 30\%) y 0. Muy baja - frecuencia $(0,1-0,9 \%)$.

DC: Demanda de comercialización que expresa el potencial de comercialización o uso de la especie; 3. Alta - muy demandada; 2. Media - medianamente demandada; 1. Baja - poco demandada y 0 . Muy baja - sin demanda.
Fase III. Análisis de datos estadísticos. Para el tratamiento estadístico de los datos se aplicó una Prueba de t Student en el software estadístico R, con un nivel de confianza del 95\% y dos grados de libertad; con ello se determinó si existe diferencias estadísticas significativas entre el conocimiento que posee cada estrato, además se correlacionaron los datos de edad, escolaridad y el número de frases. Finalmente las variables de uso y manejo fueron analizadas a través de estadística descriptiva.

\section{RESULTADOS Y DISCUSIÓN}

Características de los informantes clave. Las 10 personas o informantes clave, mujeres en su mayoría, presentan un rango de edad de 20 a 60 años y diferentes niveles de escolaridad; se caracterizan por su dedicación al hogar alternando con actividades de producción agropecuaria, entre ellas, el manejo del huerto mixto, de donde derivan su sustento. Según el Ardila (2010), en este tipo de sistemas productivos, las mujeres ven al huerto como una extensión de las actividades reproductivas. Los huertos se han constituido como un espacio de distracción y esparcimiento de las mujeres.

Características de la línea base de conocimiento. La línea base de conocimiento (Tabla 1) estuvo conformada por 1068 enunciados formales, distribuidos en 638 enunciados de atributo-valor, 342 enunciados causales, 52 enunciados comparativos y 36 enunciados de enlace; ordenados en 29 tópicos en torno a los siguientes temas: uso de las especies y manejo de los componentes del huerto. 
Tabla 1. Características de la línea base de conocimiento, vereda Concepción Alto, municipio de Pasto, Nariño.

\begin{tabular}{lc}
\hline \multicolumn{1}{c}{ Característica } & Cantidad \\
\hline Fuentes & 10 \\
Términos formales & 661 \\
Enunciados de atributo & 638 \\
Enunciados causales & 342 \\
Enunciados comparativos & 52 \\
Enunciados de enlace & 36 \\
Jerarquía de objetos & 19 \\
Tópicos & 29 \\
Número de especies mencionadas & 89 \\
\hline
\end{tabular}

El conocimiento local reportado es homogéneo debido a que presenta similitud en cuanto al manejo de términos, por ejemplo: uso de las especies y que servicios que éstas brindan. A diferencia del estudio realizado por Muñoz et al. (2008), ésta investigación muestra que los productores conocen sobre aspectos fenológicos de las especies agrícolas y forestales, no obstante, en algunos casos la época en que esto ocurre no es puntual. Sin embargo, utilizan las fases lunares como un referente para programar sus actividades agrícolas.

Diferenciación del conocimiento. Tomando como criterio el número de enunciados proporcionado por cada informante, el conocimiento entre los estratos I $(\mu=94 \pm 27)$, II $(\mu=127 \pm 22)$ y III $(\mu=75 \pm 13)$ mostró diferencias estadísticas significativas ( $p$-value $=0,3988>0,05$ ), es decir, que los estratos comparten el mismo conocimiento. El estrato II mostró mayor conocimiento con un promedio de $127 \pm 22$ frases por persona, esto significa que a medida que la edad aumenta, disminuye el número de frases mencionadas, reflejando una correlación negativa entre las variables edad y número de frases $(\mathrm{r}=-0,31)$, posiblemente se debe a que las personas de mayor edad reflejaban timidez y dificultad de comuni- cación. No obstante estas personas expresaron un conocimiento más profundo derivado de la experiencia en el trabajo agropecuario. Al respecto Johnson (1992), señala que las personas de mayor edad han acumulado notables experiencias en el manejo de cultivos, por lo tanto, su visión es más amplia que la gente joven.

Por otra parte, la escolaridad influye en la percepción de los agricultores, reflejada en el uso de términos técnicos y la profundización al momento de expresar el conocimiento en aspectos como manejo y servicios ecosistémicos de ciertas especies. No obstante, los productores que han tenido contacto directo con extensionistas o han asistido a capacitaciones, reportaron más conocimientos en cuanto a temas relacionados con bancos forrajeros, nutrición bovina con base en forraje de árboles, factores negativos y positivos asociados a la producción de leche y uso medicinal de las especies arbóreas. En este particular, el productor ha empezado a explorar y a experimentar nuevos conocimientos, gracias al apoyo y acompañamiento institucional, lo que le ha permitido de alguna forma valorarlo, retroalimentarlo y legitimarlo (Coral et al., 2011).

\section{Conocimiento local sobre el uso de las espe-} cies del huerto. Los informantes clave consideran que los huertos son un sistema productivo muy valioso dentro de la finca por los múltiples beneficios que las especies les brindan. A partir de ello, se identifica una amplia información sobre el uso de las especies, proveniente de observaciones, experiencias propias e incluso creencias que se transmiten de generación en generación. Dentro de los usos más comunes se encuentra la medicina humana y animal, las cercas vivas, los postes, la leña, la madera, la sombra, la ornamentación y la alimentación humana y animal. Lo anterior coincide con lo reportado por Muñoz et al. (2008) sobre los usos de las especies agrícolas y leñosas debido a que son especies multifuncionales. 
A continuación se destacan los usos registrados por cada uno de los componentes del huerto:

Uso medicinal. Dentro de esta categoría, se identificaron 48 especies entre agrícolas, arbóreas y arbustivas (Tabla 2). Las especies más comunes dentro del huerto son las aromáticas. De acuerdo con la cantidad de beneficios medido por el coeficiente de importancia (CI), las más representativas para el uso medicinal son: Alfalfa (Medicago sativa L.), Papa (Solanum tuberosum L.), Eucalipto (Eucalyptus globulus Labill.) y Chilca negra (Bacchiaris latifolia R\&P), con valores de CI iguales a 2,17; 2,17; 2,03 y 2 , respectivamente.

Las personas entrevistadas reafirman una vez más, que son las mujeres las que lideran el manejo del huerto, además, poseen un conocimiento amplio en propiedades medicinales de las plantas y estratégicamente, es el principal lugar donde por tradición familiar se transmite y conserva el conocimiento local (Colón, 2013).

Los usos medicinales de las plantas están relacionados con el control de problemas sanitarios en animales y enfermedades en seres humanos. Beneficios similares en cuanto al potencial medicinal de las especies leñosas, fueron encontrados por Coral et al. (2011), quienes afirman que el componente arbóreo en el ámbito medicinal tiene mucha relevancia, sin embargo, el conocimiento no alcanza mayor desarrollo, difusión y aplicación a nivel local.

Otras de las múltiples funciones que tienen las plantas medicinales como, ruda (Ruta graveolens L.), caléndula (Calendula officinalis L.) y altamisa (Ambrosia peruviana Willd.) es servir como insecticidas bioracionales.

Tabla 2. Conocimiento local sobre uso medicinal de especies más comunes en la vereda Concepción Alto, municipio de Pasto, Nariño.

\begin{tabular}{|c|c|c|c|c|}
\hline Nombre común & Nombre científico & Familia & $\mathrm{CI}^{*}$ & Atributos medicinales \\
\hline Alfalfa & Medicago sativa $\mathrm{L}$. & Mimosaceae & 2,17 & Anemia \\
\hline Papa & Solanum tuberosum L. & Solanaceae & 2,17 & Gastritis \\
\hline Eucalipto & Eucalyptus globulus Labill & Myrtaceae & 2,03 & Achaque de gallinas, resfriado \\
\hline Chilca negra & Baccharis latifolia $\mathrm{R} \& \mathrm{P}$ & Asteraceae & 2 & Dolor de rodillas, achaque de cuyes \\
\hline Zanahoria & Daucus carota L. & Apiaceae & 1,92 & Curar vista \\
\hline Remolacha & Beta vulgaris L. & Amaranthaceae & 1,88 & Anemia y purificar sangre \\
\hline Encino & Weinmannia tomentosa L.f & Cunoniaceae & 1,83 & Diarrea de ganado \\
\hline Uvilla & Physalis peruviana $\mathrm{L}$. & Solanaceae & 1,65 & Curar vista y habla de los bebes \\
\hline Haba & Vicia faba L. & Fabaceae & 1,64 & Gripa \\
\hline Caléndula & Calendula officinalis $\mathrm{L}$. & Asteraceae & 1,54 & $\begin{array}{l}\text { Previene caspa, cicatrizante, } \\
\text { desinfectante, repelente de insectos }\end{array}$ \\
\hline Manzanilla & Chamaemelum nobile (L.) All. & Asteraceae & 1,4 & Desinflamar matriz, fiebre \\
\hline Menta & Mentha sp & Lamiaceae & 1,37 & Dolor de estómago \\
\hline Sauco & Sambucus nigra L. & Adoxaceae & 1,37 & Tos \\
\hline Tomillo & Thymus vulgaris L. & Lamiaceae & 1,33 & Dolor de estómago \\
\hline Tomate de árbol & Solanum betacea (Cav). & Solanaceae & 1,31 & Paperas y dolor de garganta \\
\hline Orégano & Origanum vulgare L. & Lamiaceae & 1,28 & $\begin{array}{l}\text { Dolor de estómago, dieta de } \\
\text { embarazo }\end{array}$ \\
\hline Hierba buena & Mentha x piperita L. & Lamiaceae & 1,21 & Desparasitante \\
\hline
\end{tabular}




\begin{tabular}{lllcl}
\hline Nombre común & \multicolumn{1}{c}{ Nombre científico } & \multicolumn{1}{c}{ Familia } & CI* & \multicolumn{1}{c}{ Atributos medicinales } \\
\hline Ruda & Ruta graveolens $\mathrm{L}$. & Rutaceae & 1,17 & $\begin{array}{l}\text { Cólicos menstruales y repelente } \\
\text { de insectos }\end{array}$ \\
\hline Jíquima & $\begin{array}{l}\text { Smallanthus sonchifolius } \\
\text { (Poepp.) H. Rob. }\end{array}$ & Asteraceae & 1,08 & Diabetes \\
\hline Poleo & Mentha pulegium L. & Lamiaceae & 0,92 & Dolor estómago, diarrea \\
\hline Toronjil & Melissa officinalis L. & Lamiaceae & 0,83 & Controlar nervios \\
\hline Malvalta & Lavatera arborea L. & Malvaceae & 0,67 & Fiebre \\
\hline Geranio & Pelargonium peltatum L. & Geraniaceae & 0,5 & Dolor de muelas \\
\hline Valeriana & Valeriana officinalis L. & Caprifoliaceae & 0,5 & Controlar nervios \\
\hline
\end{tabular}

$\mathrm{CI}^{*}$ : Coeficiente de Importancia.

Uso alimenticio. Se reportaron 42 especies comestibles, consideradas como la base principal de su alimentación (Tabla 3). Estos registros son menores a lo reportado por García et al. (2008), quienes encontraron 104 especies comestibles en un estudio realizado en la cuenca media del río Guayas - Ecuador; así mismo, Álvarez (2014), encontró 21 especies promisorias de uso alimenticio en el corregimiento de San Francisco de Asís, Acandí- Chocó. A nivel local Montenegro et al. (2017), en un estudio sobre agrodiversidad de los huertos caseros en la región andina del sur de Colombia, afirman que en un huerto tipo, de 142 especies encontradas, el 35\% correspondía a especies agrícolas. En la presente investigación, del total de las especies obtenidas, diez fueron priorizadas como las más importantes con valores de CI iguales a Vicia faba L. (1), Beta vulgaris L. (1,3), Pisum sativum L. $(1,4)$, Brassica oleracea var. Botrytis L. (1,4), Zea mayz L. $(1,6)$, Ullucus tuberosus Caldas. $(1,7)$, Allium fitolosum L. (1,8), Brassica oleracea L. $(1,9)$, Daucus carota L. $(1,9)$ y Solanum tuberosum L. $(2,2)$.

Tabla 3. Conocimiento local de especies alimenticias más representativas en la vereda Concepción Alto, municipio de Pasto, Nariño

\begin{tabular}{|c|c|c|c|c|c|}
\hline $\begin{array}{l}\text { Nombre } \\
\text { común }\end{array}$ & $\begin{array}{l}\text { Nombre } \\
\text { científico }\end{array}$ & Familia & $\mathrm{CI}^{*}$ & $\begin{array}{c}\text { Parte } \\
\text { utilizada }\end{array}$ & $\begin{array}{c}\text { Modo de } \\
\text { preparación }\end{array}$ \\
\hline Haba & Vicia faba $\mathrm{L}$. & Fabaceae & 1 & Fruto & Tostadas y sopas \\
\hline Acelga & Beta vulgaris L. & Amarantaceae & 1,3 & Hojas & Guisadas \\
\hline Arveja & Pisum sativum L. & Fabaceae & 1,4 & Fruto & Sopas y guisos \\
\hline Coliflor & Brassica oleracea var.Botrytis L. & Brassicaceae & 1,4 & $\begin{array}{l}\text { Inflorescencia y } \\
\text { hojas }\end{array}$ & Guisadas, ensaladas y cremas \\
\hline Maíz & Zea mayz L. & Poaceae & 1,6 & Fruto & Sopas \\
\hline Olluco & Ullucus tuberosus Caldas & Basellaceae & 1,7 & Tubérculo & Guisadas y sopas \\
\hline Cebolla & Allium fitolosum $\mathrm{L}$. & Amaryllidaceae & 1,8 & Bulbo & Guiso, sopa y ensalada \\
\hline Repollo & Brassica oleracea L. & Brassicaceae & 1,9 & Hojas & Ensaladas y sopas \\
\hline Zanahoria & Daucus carota L. & Apiaceae & 1,9 & Raíz & Jugos, guisadas, cremas y ensaladas \\
\hline Papa & Solanum tuberosum $\mathrm{L}$. & Solanaceae & 2,2 & Tubérculo & Guisadas, ensaladas, sopas y fritas \\
\hline
\end{tabular}

CI* Coeficiente de Importancia. 
A pesar que las especies medicinales superan en número a las alimenticias por su facilidad de manejo al cultivarlas, sigue siendo prioridad para los campesinos en la seguridad alimentaria. Esta misma importancia la resaltan Traversa et al. (2000) quienes señalan que el objetivo principal de los huertos tradicionales es la búsqueda de la autosuficiencia alimenticia y que la frecuencia de especies por uso es el indicador más adecuado para conocer el objetivo principal de un huerto.

Es de resaltar, que dentro del huerto se han propagado especies nativas de importancia alimenticia y cultural que han sido rescatadas durante el tiempo, como por ejemplo la cebolla (A. fitolosum), repollo o malque (B. oleracea) y algunas variedades de papa (S. tuberosum), este hecho permite conservar la diversidad genética en la zona. Al respecto, Montagnini et al. (2015) señalan la importancia de los huertos caseros como bancos genéticos de cultivos antiguos y fuentes de investigación para nuevas variedades cultivadas.

Uso forrajero. Se identificaron 21 especies forrajeras, de las cuales, cuatro son de mayor preferencia por el ganado, entre las que se encuentran: Aliso (Alnus acuminata Kunth.), Sauco (Sambucus nigra L.), Alfalfa (M. sativa L.), Trébol (Trifolium repens L.). Estas especies poseen altos contenido de proteína y fibra que contribuyen al engorde y a mejorar la calidad de la leche, principalmente las dos últimas especies (Peters, 2010). Al respecto, argumentos empíricos destacan la importancia del aliso "la hoja de aliso sirve para colocarle al ganado cuando esta escasa la hierba y en segundo lugar la hoja se la utiliza cuando está seca para realizar bloques nutricionales para los cuyes". Por otra parte, Joya et al. (2004) y Vásquez et al. (2014), utilizaron criterios como, abundante productividad de hojas, alta palatabilidad, olor a miel, abundante proteína y buenas productoras de leche, para clasificar las plantas como buenos forrajes.

Fue común que las personas entrevistadas destacaran los múltiples beneficios del Aliso (A. acuminata Kunth.), entre ellos, mejorar la producción de pasto por su aporte de nitrógeno al suelo, además de proporcionar sombra y protección contra lluvia y fuertes vientos (Figura 1). Al respecto, Insuasty et al. (2011) afirman que el pasto kikuyo (P. clandestinum) en un sistema silvopastoril (SSP) con $A$. acuminata, mejoró algunas características agronómicas como producción de forraje verde, producción de materia seca y altura del pasto, comparativamente con la pradera en monocultivo.

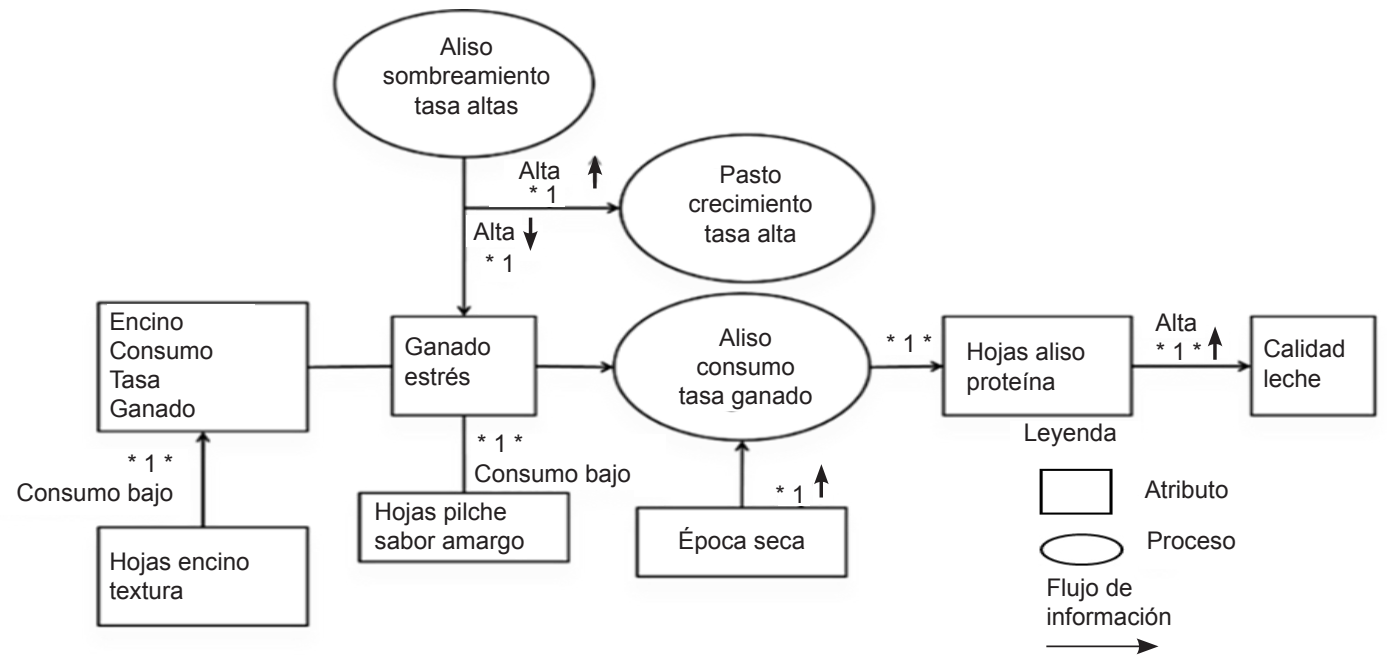

Figura 1. Conocimiento local de especies forrajeras en la vereda Concepción Alto, municipio de Pasto, Nariño. 
Otros usos. Dentro de las especies forestales mencionadas por los informantes, 18 se destacan por su valor y uso dendroenergético (Tabla 4), recurriendo a estas como única fuente, por cuanto los costos que demanda el uso de energía eléctrica y gas metano no está al alcance de sus ingresos. Esto ha ocasionado que muchas especies nativas como Manduro (Clethra fagifolia Kunth), Encino (W. tomentosa L.f), Amarillo (Miconia theaezans Bonpl.) Cogn.) y Arrayan (Myrcianthes rhopaloides (Kunth) McVaugh) sean sobreexplotadas por su alto poder calorífico, baja producción de ceniza, baja producción de humo y alta producción de carbón. Estas mismas características les atribuyen los ganaderos en Cañas, Costa Rica, al momento de hacer la selección de las especies forestales utilizadas para leña (Muñoz, 2006).

La madera es otro uso reportado, donde las especies son utilizadas para construcción de casas, corrales y muebles. En total se mencionaron 11 especies, de las cuales Pilche (P. murris), Manduro (C. fagifolia) y Encino (W. tomentosa) son consideradas de mejor calidad. El Eucalipto
(E. globulus) a pesar de no ser considerada una madera muy fina, es de uso común en la zona por su rápido desarrollo y fácil manejo. Muñoz et al. (2013) encontraron que el mayor uso que le dan los agricultores a los árboles que se incorporan a las fincas, es para leña y madera, principalmente de las especies nativas.

Los servicios ambientales que pueden proporcionar estos sistemas como sombra, regulación hídrica, mejoramiento de suelos y biodiversidad son poco conocidos o valorados, sin embargo, como se muestra en la Figura 2, se destaca que los árboles mediante la descomposición de sus hojas incrementan la humedad y fertilidad del suelo. Especies como Aliso (A. acuminata), Pucasacha (Tibouchina mollis (Bonpl.) Cogn.) y Acacia (A. melanoxylon) son consideras benéficas para el suelo. Insuasty et al. (2011) señalan que el Aliso (A. acuminata) sin ser una leguminosa, es capaz de realizar un proceso de fijación de nitrógeno atmosférico mediante el actinomiceto del género Frankia, el cual beneficia a todo el sistema aportando nutrientes y creando un ambiente favorable.

Tabla 4. Conocimiento local de especies forestales más representativas, en la vereda Concepción Alto, municipio de Pasto, Nariño.

\begin{tabular}{llcccc}
\hline \multicolumn{1}{c}{$\begin{array}{c}\text { Nombre } \\
\text { Común }\end{array}$} & \multicolumn{1}{c}{$\begin{array}{c}\text { Nombre } \\
\text { Científico }\end{array}$} & $\begin{array}{c}\text { Producción } \\
\text { de ceniza }\end{array}$ & $\begin{array}{c}\text { Producción } \\
\text { de humo }\end{array}$ & $\begin{array}{c}\text { Producción } \\
\text { de brasa }\end{array}$ & $\begin{array}{c}\text { Tasa de } \\
\text { combustión }\end{array}$ \\
\hline Arrayan & $\begin{array}{l}\text { Myrcianthes rhopaloides } \\
\text { (Kunth) McVaugh }\end{array}$ & Baja & Baja & Alta & \\
Colla negra & Samallanthus pyramidalis & Baja & Baja & Baja & Rápida \\
Cedrillo & Brunnelia sp & Alta & Alta & Baja & Rápida \\
Aliso & Alnus acuminata Kunth & Alta & Alta & Baja & Rápida \\
Eucalipto & Eucalyptus globulus Labill & Alta & Media & Baja & \\
Pilche & Prunus muris Cuatrec. & Baja & Baja & Alta & Lenta \\
Acacia & Acacia melanoxylon R. Brown & Media & Media & Baja & Rápida \\
Manduro & Clethra fagifolia Kunth & Baja & Baja & Alta & Lenta \\
Encino & Weinmannia tomentosa L.f & Baja & Baja & Alta & Lenta \\
Chilca negra & Baccharis latifolia R\&P & Baja & Baja & Baja & Lenta \\
Capulí & Prunus serotina Ehrhart & Baja & Baja & Alta & Lenta \\
Charmolan & Policourea $s p$ & Media & Media & Media & Lenta \\
\hline
\end{tabular}




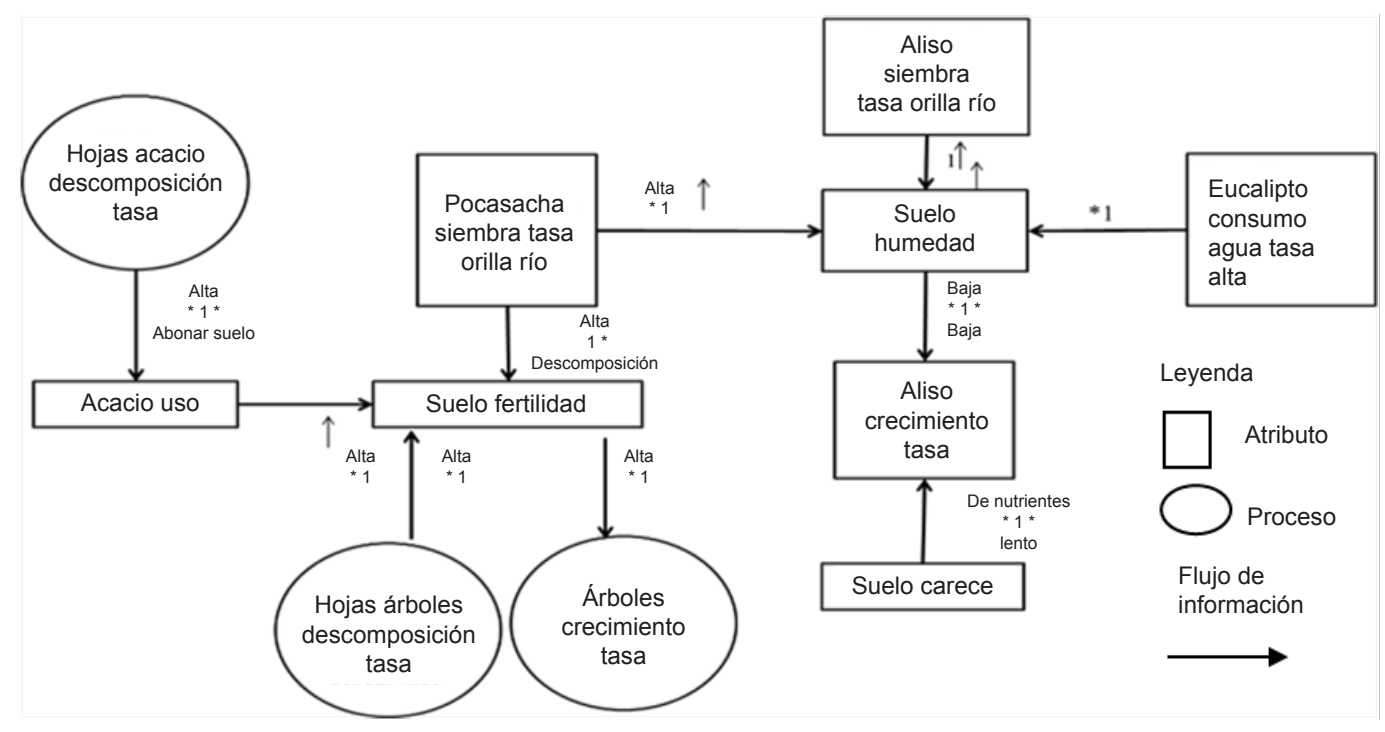

Figura 2. Conocimiento local interacción árbol-suelo en los huertos mixtos vereda Concepción Alto, municipio de Pasto, Nariño.

Por otro lado, las especies Aliso (A. acuminata), Moquillo ( $S$. brachybotrys Turcz), Manduro (C. fagifolia), Arrayan (M. rhopaloides) y Quillotocto (T. stanns (L.) Juss.ex Kunth), se destacan por la protección y conservación de las fuentes hídricas, razón por el cual se evita la tala de estos árboles y promueven su siembra. En otro estudio realizado en la misma zona Vela y Jiménez (2009) encontraron que los campesinos siembran especies nativas en sus fincas para la regulación hidrológica, protección de zonas de recarga, estabilización del clima, entre otros. Por el contrario, especies como el eucalipto (E. globulus), acacia (A. melanoxylon), urapán (F. chinnensis Roxb.) y pino (P. patula Schltdl. \& Cham.) no se siembran cerca de las fuentes hídricas, debido a que sus raíces consumen mucha agua.

En cuanto a la sombra, especies como el capulí (P.serótina), sauco (S.nigra), chilca negra (B. latifolia), aliso (A.acuminata) y acacia (A. melanoxylon), protegen a los cultivos y al ganado del estrés producido por la radiación solar. Particularmente el aliso (A. acuminata) es muy útil durante la época seca debido a que proporciona una sombra fresca debido a la amplitud de su follaje; al respecto Muñoz (2006) afirma que los ganaderos de Cañas, Costa Rica, hacen alusión a las sombras frescas, como aquellas provenientes de especies con gran cantidad de hojas y ramas que le permiten formar sombras densas y frescas.

Es destacable en la zona, el uso de los árboles como cercas vivas, los que brindan entre otros servicios el control de la intensidad del frío y la brisa, proteger al ganado del estrés, mantener la humedad en el suelo, reemplazar los postes muertos y contribuir a la biodiversidad. Dentro delas especies usadas para este fin se mencionan, el Aliso (A. acuminata), Sauco (S. nigra), Cedrillo (Brunnellia sp), Chilca (B. latifolia), Acacia (A. melanoxylon) y Colla negra (S. pyramidalis). Corroborando lo anterior, Vela y Jiménez (2009) encontraron que para los productores las cercas vivas son de mayor importancia, por cuanto ellas "ayudan a conservar el agua y mantenerla en época seca", protección y conservación de suelos y sobre todo representan baja inversión para su establecimiento, sin contar los múltiples beneficios socioeconómicos. 
Manejo del huerto. El manejo de las especies agrícolas y arbóreas es netamente convencional. Está acompañado de prácticas culturales y el uso de productos químicos. Aunque, el interés que tiene la comunidad en adquirir nuevos conocimientos, ha permitido implementar nuevas prácticas sostenibles, por ejemplo, la producción y uso de abono orgánico, bioinsumos y prácticas agroecológicas. En este particular la Ardila (2010), destaca que la transferencia y recreación de nuevos conocimientos con las familias campesinas se da en un proceso bidireccional en el propio huerto, que es espacio de experimentación, confrontación y análisis. A partir de ello, los campesinos incorporan a su experiencia prácticas que les sirven y desechan lo que dificulta o no funciona, así los conocimientos se integran en una dinámica de aprender haciendo.

Parte del conocimiento que se tiene sobre el manejo de los huertos mixtos, provienentambién de lo trasmitido generacionalmente y por la experiencia vivenciada día tras día. En el caso de las hortalizas, las comunidades recomiendan no sembrar directamente sino a través de semilleros, esto garantiza la sobrevivencia y buena producción. Aguirre (2013) señala que en las hortalizas que requieren trasplante, la elaboración y eficaz manejo de los semilleros son, en gran medida, esenciales para obtener una excelente cosecha.

Dentro de esta investigación se resalta que la comunidad basa la producción en la influencia de la luna sobre los cultivos y árboles, afirmando que especies como el Romero (Rosmarinus officinalis L.), Haba (V. faba), Lechuga (L. sativa), Aliso (A. acuminata), Sauco ( $S$. nigra), Papa (S. tuberosum), Repollo (B. oleracea), Espinaca (S. oleracea) y Maíz (Z. mays), se deben sembrar en cuarto menguante para estimular el desarrollo y crecimiento. La observación de la luna, como medio para decidir el inicio de las labores agrícolas es característica tradicional en muchas regiones, por ejemplo, para los agricultores en Puebla, México la luna llena ("recia") es ideal para iniciar la siembra. Al hacerlo se espera que las plantas presenten menos plagas, mayor vigor y alta producción (Miranda et al., 2009).

El riego fue otra de las prácticas de manejo mencionadas. Las hortalizas (lechuga, coliflor, brócoli y acelga) son las que mayor demanda de agua requieren. Al respecto, Úbeda y Moreno (2015), manifiestan que estas especies necesitan que en todo momento se mantenga el suelo en un estado óptimo de humedad, para tener un buen desarrollo vegetativo (raíz, tallo, hojas, fruto), debido a las características de su masa foliar.

Para la fertilización de los cultivos se ha generalizado el uso de abono orgánico, por ejemplo los cultivos de cebolla, coliflor, acelga y brócoli son fertilizados con excretas de cuy aportando nutrientes que estimulan el desarrollo de estos cultivos. También se registró el uso de bioinsumos provenientes de especies como la ruda, cebolla, altamisa, ajo y ají. Para Altieri y Toledo (2010), muchos agricultores enriquecen sus suelos juntando materiales y nutrientes como son abonos orgánicos, desperdicios forestales, entre otros, así como el uso de insecticidas botánicos y/o repelentes para minimizar la incidencia de plagas. Con este tipo de prácticas permiten desarrollar agroecosistemas más sustentables.

En cuanto al manejo de las especies arbóreas sólo se realizan podas, principalmente a especies como la acacia (A. melanoxylon), aliso (A. acuminata), cedrillo (Brunnellia $s p$ ), dicha práctica se realiza en luna llena favoreciendo el crecimiento. Los resultados similares encontró Muñoz (2006), donde los agricultores, según sus creencias y conocimientos heredados de los ancestros, afirman que el apolillamiento de la madera está relacionado con las fases lunares. 


\section{CONCLUSIONES}

Los informantes clave seleccionados para esta investigación fueron mujeres en su mayoría de 20 años en adelante, quienes han liderado la producción y el manejo del huerto mixto a nivel local, visibilizando el trabajo reproductivo de las mujeres y el aporte directo que ellas hacen a la seguridad alimentaria familiar. Las mujeres consideran al huerto como el espacio de creación y recreación del conocimiento, saberes, prácticas, entre otros, esto ha generado una valoración de del rol que cumple en la familia y en la sociedad.

Los huertos mixtos presentes en el la Vereda Concepción Alto, son sistemas productivos de alta diversidad de especies, 89 en total, entre agrícolas, arbóreas y arbustivas, alguna de ellas nativas, de alta importancia alimenticia y cultural que brindan múltiples beneficios a las comunidades que las practican, de ahí que se siguen reproduciendo de generación en generación, incluyendo cada días más especies a este tipo de agroecosistemas.

De acuerdo al Coeficiente de Importancia, los productores de la vereda Concepción Alto, consideran que las especies de mayor importancia son Papa (Solanum tuberosum L.), Alfalfa (Medicago sativa L.) y Aliso (Alnus acuminata K), que tienen fácil adaptación a las condiciones de la zona, fácil manejo y son multifuncionales, proporcionando diferentes beneficios socioculturales.

La inclusión del árbol dentro del huerto mixto ha representado múltiples beneficios no solo a nivel ambiental por su aporte de materia orgánica, regulación hídrica, protección contra vientos y fijación de nitrógeno de algunas especies, sino por los beneficios a la familia principalmente leña, madera, frutos comestibles, forraje, medicina, entre otros.

Conflicto de intereses: Los autores declaran que no hay conflicto de interés.

\section{REFERENCIAS BIBLIOGRÁFICAS}

Aguirre, S. (2013). Horticultura. Bogotá: Universidad Nacional Abierta y a Distancia - UNAD. 241p.

Altieri, M y Toledo, V. (2010). La Revolución agroecológica de América Latina: Rescatar la naturaleza, asegurar la soberanía alimentaria y empoderar al campesino. Revista El Otro Derecho. 48: 163-202.

Álvarez, L. (2014). Plantas promisorias de uso alimenticio del Darién, Caribe colombiano. Boletín de Antropología. 29 (48):41-65. doi: 10.17533/ udea.boan.v29n48a02.

Ardila, J. (2010). Extensión rural para el desarrollo de la agricultura y la seguridad alimentaria: aspectos conceptuales, situación y una visión de futuro. San José s Costa Rica: Instituto Interamericano de Cooperación para la Agricultura. 128p.

Bentes, M. \& Gama, J. (1999). Huertos caseros en la comunidad ribereña de Villa Cuera, en el municipio de Brangança en el Noroeste Parense Brasil. Rev. Agroforest. 24: 8-13.

Colón, A. (2013). Conocimiento local sobre plantas medicinales en tres comunidades del municipio de Santa Fe, Colón. Recuperado de https:// patriciacolon.files.wordpress.com/2010/09/ primer-borrador-informe-de-investigacic3b3n. pdf. 38 p.

Coral, D., Coral, J. \& Muñoz, D. (2011). Caracterización del conocimiento local del componente arbóreo en fincas ganaderas. Revista de Ciencias Agrícolas. 28 (2): 18-30.

CORPONARIÑO - Corporación Autónoma Regional de Nariño. (2012). Plan de acción institucional 2012 - 2015. Recuperado de http://corponarino. gov.co/expedientes/planeacion/pai20122015/ pai20122015f.pdf. 45-46 p.

Dixon, H., Doores, J., Joshi, L. \& Sinclair, F. (2001). Kit de herramientas de conocimiento agroforestal (AKT5). Bangor: Facultad de Ciencias Forestales y Agricultura, Universidad de Gales. 171p.

Farrington \& Martín. (1988). Conocimiento tradicional de las comunidades campesinas. Recuperado de http://www.odi.org/sites/odi. org.uk/files/odi-assets/publications-opinionfiles/8136.pdf 
García, L., Suatunce, P. \& Torres, T. (2008). Plantas útiles en los sistemas agroforestales tradicionales del litoral ecuatoriano. Revista de Ciencia y Tecnología. 1(2): 65-71.

Insuasty, E., Apráez, J. \& Navia, F. (2011). Efecto del arreglo silvopastoril aliso (AlnusAcuminata K.) y kikuyo (Pennisetum Clandestinum H.) sobre el comportamiento productivo en novillas Holstein en el altiplano del departamento de Nariño. Agroforestería Neotropical. 1(1): 29-36.

Johnson, M. (1992). Reconociendo el valor del conocimiento tradicional. CIID Informa. 21(1): 10-13.

Joya, M., López, M., Gómez, R. \& Harvey, C. (2004). Conocimiento local sobre el uso y manejo de los árboles en las fincas ganaderas del municipio de Belén, Rivas. Revista Encuentro. 68: 44 - 59.

Lok, R. (1998). Introducción a los huertos caseros tradicionales tropicales. Turrialba, Costa Rica: CATIE. Proyecto Agroforestal CATIE/GTZ. 157 p.

Miranda, J., Herrera, B., Paredes, J. \& Delgado, A. (2009). Conocimiento tradicional sobre predictores climáticos en la agricultura de los llanos de Serdán, Puebla, México. Revista Tropical and Subtropical Agroecosystems. 10 (2): 151-160.

Montagnini, F., Somarriba, E., Murgueitio, E., Fassola, H. \& Eibl, B. (2015). Sistemas Agroforestales: Funciones productivas, socioeconómicas $y$ ambientales. Serie técnica. Informe técnico 402. Catie, Turrialba, Costa Rica: Editorial CIPAV. 454p.

Montenegro, M., Lagos, T. \& Velez, J. (2017). Agrodiversidad de los huertos caseros de la región andina del sur de Colombia. Revista de Ciencias Agrícolas. 34 (1): 50-63. doi: 10.22267/ rcia.163302.53.

Muñoz, D., Harvey, C., Sinclair, F., Mora, J. \& Ibrahim, M. (2003). Conocimiento local de la cobertura arbórea en sistemas de producción ganadera en dos localidades de Costa Rica. Revista Agroforesteria en las Américas. 10(39-40): 61-68.

Muñoz, D. (2006). Conocimiento local de la cobertura arbórea en sistemas de producción ganadera en dos localidades de Costa Rica. Revista de Ciencias Agrícolas. 23(1-2): 155-170.
Muñoz, D., España, M. \& Obregón, L. (2008). Conocimiento local sobre el uso y manejo de las especies arbóreas y agrícolas en huertos mixtos, en dos localidades del municipio de Tumaco, departamento de Nariño. Revista de Ciencias Agrícolas. 25 (1): 63-76.

Muñoz, D., Calvache, D. \& Yela, J. (2013). Especies forestales con potencial agroforestal para las zonas altas en el departamento de Nariño. Revista de Ciencias Agrícolas. 30(1): 38-53.

Nair, P. \& Kumar, B. (2006). Introducción, pp. 1-10. En: Nair, P. y Kumar, B. Tropical homegardens: A time-tested example of sustainable agroforestry. Dordrecht: Springer. 378p.

Peters, M., Franco, L., Schmidt, A. \& Hincapié, B. (2010). Especies forrajeras multipropósito, opciones para productores del trópico Americano. Cali: CIAT. 222p.

Sablayrolles, M. \& Andrade, L. (2009). Entre sabores, aromas e saberes: a importancia dos quintais agroflorestais para agricultores ribeirinhos no Tapajós-PA. Recuperado de http://www.sct. embrapa.br/cdagro/tema03/03tema19.pdf

Sinclair, F. \& Walker, D. (1999). Un enfoque utilitario a la incorporación de los conocimientos locales en la investigación agroforestal y la extensión. En: Buck, L., Lassoie, J. Fernandes, ECM. pp. 245275. Agroforestería sistema agrícola sustentable. Boca Ratón: LLC.

Úbeda, J. \& Moreno, A. (2015). Guía para huertos urbanos. Cultivos hortícolas. Primera edición. España: Servicio de Medio Ambiente. 102p.

Vásquez, F., Mora, J. \& Aguilar, M. (2014). Saber popular de especies forrajeras en la zona central de Nicaragua: un estudio en grupos focales. Revista de Ciencias Agrícolas. 31(1): 27-41.

Vela, M. \& Jiménez, F. (2009). Sistemas agroforestales en áreas de recarga de agua para consumo humano en la cuenca alta del río Bobo, Colombia. Recuperado de http://bco.catie. ac.cr/portal-revistas/index.php/RRNA/article/ view/121/224

Traversa, T., Fierros, A., Gómez, M., Leyva, J. \& Hernández, R. (2000). Los huertos caseros de Zaachila en Oaxaca, México. Revista Agroforestería de las Américas. 7 (28): 12-15. 